\title{
Measurements of effective porosity of pharmaceutical tablets using THz TDS
}

\author{
Iliya Tikhomirov ${ }^{1}$, Daniel Markl ${ }^{2}$, and Mira Naftaly ${ }^{1}$ \\ ${ }^{1}$ National Physical Laboratory, Teddington TW11 0LW, UK \\ ${ }^{2}$ University of Strathclyde, Glasgow, UK
}

\begin{abstract}
The pharmaceutical industry requires a rapid nondestructive technique for monitoring porosity of tablets. Here effective porosity of compressed lactose pellets was investigated using THz time-domain spectroscopy (THz TDS).
\end{abstract}

\section{INTRODUCTION}

$\mathrm{T}$ he pharmaceutical industry is a particularly promising target area for $\mathrm{THz}$ applications, both as a spectroscopic tool and for purposes of condition monitoring (CM) and nondestructive testing (NDT). There are three main reasons for this. First, pharmaceutical materials are (semi-) transparent at $\mathrm{THz}$ frequencies and possess characteristic spectral signatures, whereas they are opaque in the visible and nearinfrared. Second, high-precision quality monitoring is of immense importance in pharmaceutical production. And third, pharmaceuticals are high-value products where increased product consistency and reduced wastage bring significant cost benefits, therefore justifying investment in relatively expensive new technologies that improve CM and NDT.

A particularly promising application of $\mathrm{THz}$ is in monitoring granularity and porosity of tablets in order to control their dissolution rate. Oral solid dosage forms, or tablets, are manufactured by compacting a blend of active pharmaceutical ingredient (API) and excipient particles. The physical and chemical properties of both API and excipient particles dominate the tableting and delivery functionality, specifically the disintegration and dissolution processes. The effective pores (i.e. open pores) in a tablet - their size and connectivity - directly affect the rate at which the physiological fluids enter the tablet, leading to swelling of the particles and eventually causing the break-up of the compact into smaller agglomerates [1]. Despite the widely recognized importance of monitoring the tablet porosity during the manufacturing process, there are no continuous in-line nondestructive techniques for achieving this. $\mathrm{THz}$ technology is a highly promising solution to this urgent problem.

A number of studies have analyzed the relationship between $\mathrm{THz}$ transmission properties of a granular pharmaceutical material and its grain size and/or porosity, reviewed in [2]. $\mathrm{THz}$ spectroscopy was also used to investigate the relationship between porosity and tablet dissolution [3].

\section{RESUlts}

In this study THz TDS was used to measure porosity of compressed lactose pellets; and furthermore, to gain additional insight into the effective porosity by soaking pellets in THztransparent index-matching fluid. D-lactose monohydrate was chosen for the pellets because it is a common excipient in pharmaceutical tablets, and therefore any results will have a direct relevance to the pharmaceutical industry. Pellets were produced by a screw press where the pressure was determined by setting the applied torque, and their porosity was measured by a $\mathrm{He}$ pycnometer. The index-matching medium was paraffin oil, which does not dissolve or react with lactose. Fig. 1a presents the loss (combining absorption and scattering) of a pellet with 0.17 porosity before and after soaking in paraffin; the inset shows the reduction of loss (at 2.2. THz) after soaking vs porosity. Fig. $1 \mathrm{~b}$ plots the increase in the refractive index (at $1 \mathrm{THz}$ ) after soaking vs porosity, together with a simple linear mixing model, showing that the index increment exceeds the model. It is seen that both loss coefficient difference and refractive index (RI) difference increase monotonically and regularly with porosity. The loss decreased from the dry to the soaked tablets at high terahertz frequencies, which can be attributes to a reduction of the scattering loss through the index-matching medium. The increase in the loss coefficient difference thus reflects an increase of effective pore size with increasing porosity. A lower compaction pressure thus does not only cause an increase in the overall porosity but also an enlargement of the effective pores. This approach therefore enables the investigation of the effective porosity by THz-TDS with an aid of an index-matching medium.
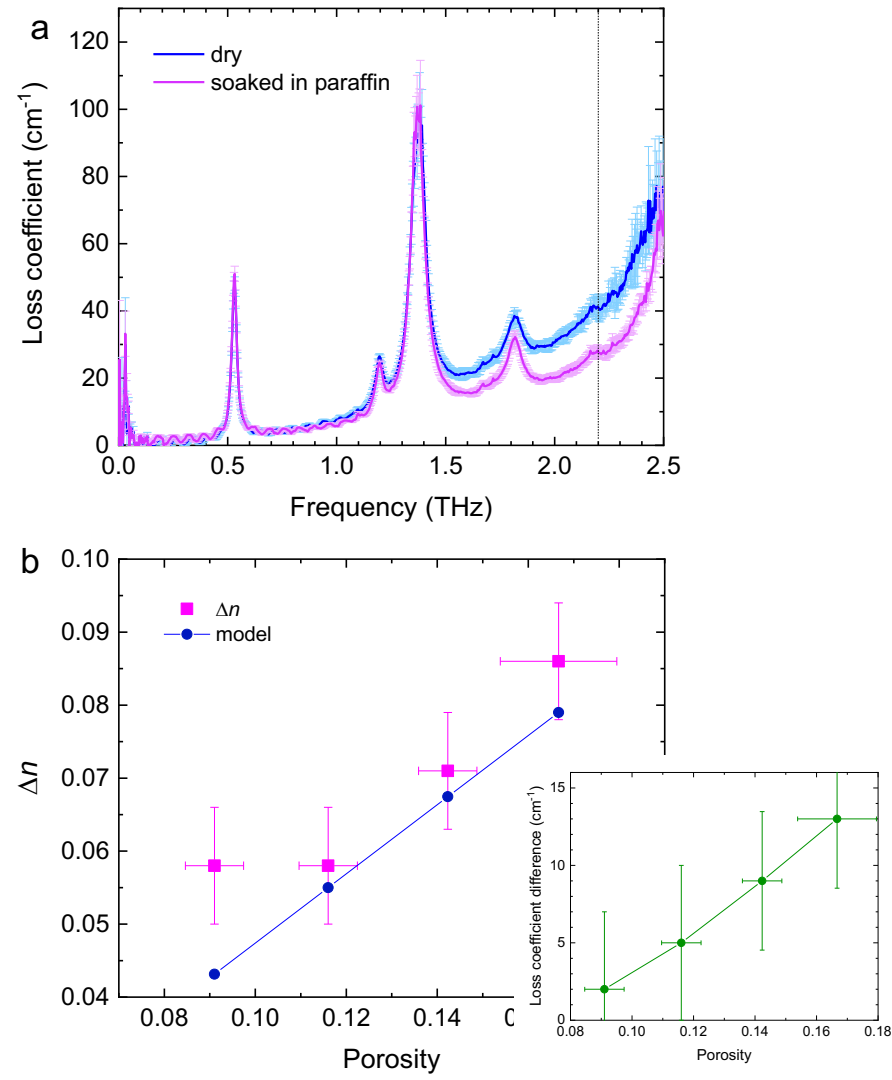

Fig. 1. (a) Loss coefficient of the pellet with 0.17 porosity before and after soaking in paraffin oil (error bars shaded). Inset: Reduction in loss (at $2.2 \mathrm{THz}$ ) after soaking, vs porosity. (b) Refractive index increase (at $1 \mathrm{THz}$ ) 
after soaking, vs porosity, together with a linear mixing model and using a $\mathrm{THz}$ refractive index of paraffin of 1.47

The aim of measuring effective porosity is to relate it to the tablet disintegration time (DT) in aqueous solution. Disintegration testing is standard practice in the pharmaceutical industry to assess that tablets meet the requirements of the relevant pharmacopoeia. To investigate this relationship, the disintegration time of the pellets was measured in water at $37^{\circ} \mathrm{C}$ by an Erweka ZT31 Disintegration Tester. As expected, disintegration time drops steeply with increasing porosity (Fig. 2.). The larger the pore space the faster the liquid uptake of the tablet. In case of lactose tablets, a faster liquid uptake accelerates the break-up of the interparticulate bonds resulting in a shorter disintegration time.

The relationship between the disintegration time and $\mathrm{THz}$ transmission properties is of particular interest. Fig. 3 plots the refractive indices of dry and paraffin-soaked pellets as a function of their disintegration time; the inset shows the difference between them. Although both refractive indices rise with the disintegration time - because both RI and DT increase as porosity decreases - the difference between the two RIs saturates beyond a DT of $10 \mathrm{~s}$. In contrast, the loss coefficient difference between dry and paraffin-soaked pellets, plotted in Fig. 4 vs disintegration time, does not reach saturation. The loss difference provides a good correlation with DT for these lactose tablets and it therefore could be used as a measure of the disintegration performance.

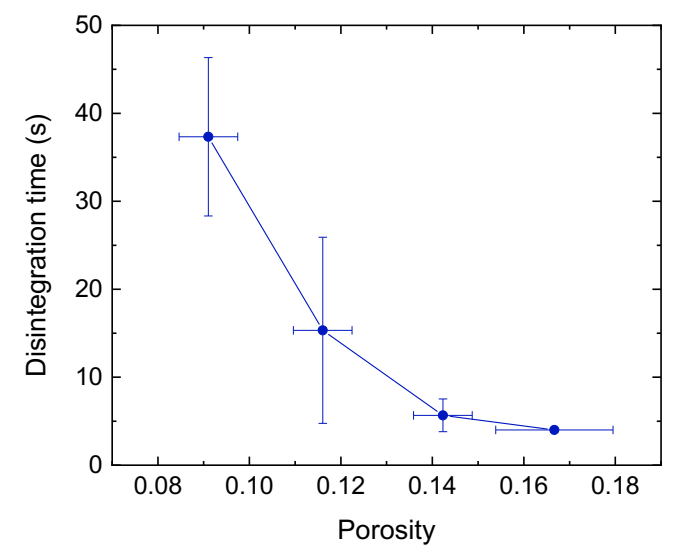

Fig. 2. Disintegration time vs porosity of the pellets.

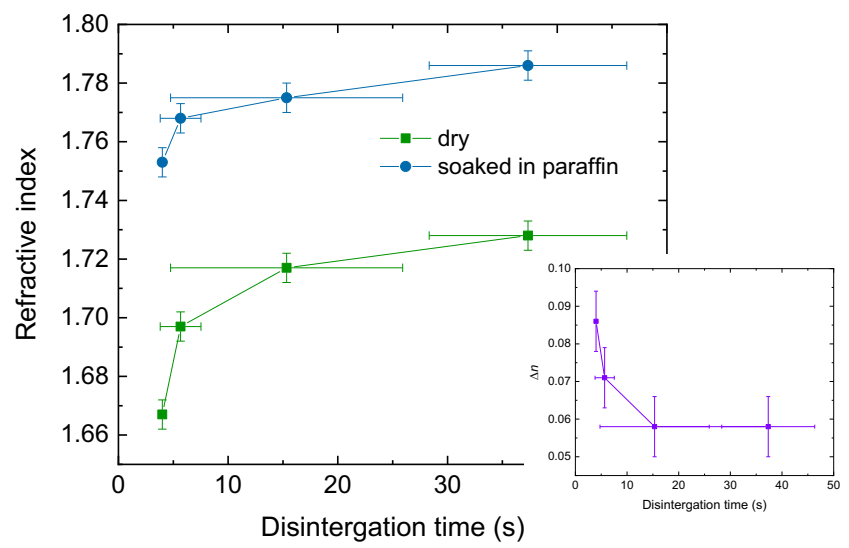

Fig. 3. Refractive indices of dry and paraffin-soaked pellets vs disintegration time. Inset: Refractive index difference, $\Delta n$, vs disintegration time.

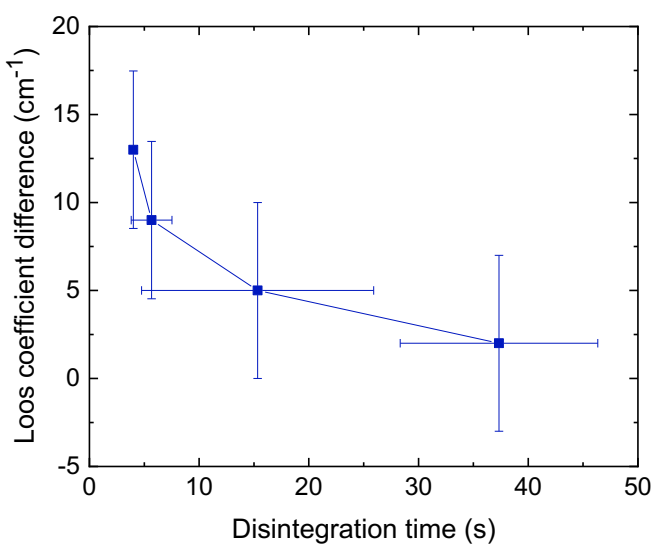

Fig. 4. Loss coefficient difference between dry and paraffin-soaked pellets vs their disintegration time.

\section{CONCLUSIONS}

Disintegration time measurements are destructive and time consuming, whereas loss difference can be measured nondestructively within a few minutes. The presented approach offers a promising technique to determine the effective porosity, which can be used to predict the DT of pharmaceutical tables non-destructively and relatively quickly.

\section{ACKNOWLEDGEMENT}

This project has received funding from the European Union's Horizon 2020 research and innovation programme under the Marie Skłodowska-Curie grant agreement No 765426 (TeraApps).

\section{REFERENCES}

[1] Markl, D. \& Zeitler, J. A., "A Review of Disintegration Mechanisms and Measurement Techniques". Pharm Res 34, 890-917 (2017).

[2] Markl, D., et al., "Characterisation of pore structures of pharmaceutical tablets: A review". Int J Pharm 538, 188-214 (2018).

[3] Mark1, D., Sauerwein, J., Goodwin, D. J., van den Ban, S., \& Zeitler, J. A., "Non-destructive determination of disintegration time and dissolution in immediate release tablets by terahertz transmission measurements". Pharm Res, 34, 1012-1022 (2017). 